\title{
Long non-coding RNA SOX21-AS1 modulates breast cancer stem cells properties and carcinogenesis via targeting SOX2
}

\author{
Zhen-Duo Lu' ${ }^{1}$, De-Chuang Jiao', Jiang-Hua Qiao ${ }^{1}$, Sen Yang ${ }^{2}$ and Zhen-Zhen Liu ${ }^{1}$ \\ ${ }^{1}$ Department of Breast surgery, Breast Cancer Center, Affiliated Cancer Hospital of Zhengzhou University, Henan Cancer \\ Hospital, Zhengzhou, Henan 450008, P.R. China \\ ${ }^{2}$ China-US (Henan) Hormel Cancer Institute, Affiliated Cancer Hospital of Zhengzhou University, Henan Cancer Hospital, \\ Zhengzhou, Henan 450008, P.R. China
}

Correspondence to: Zhen-Zhen Liv, email: liuzhenzhen_doc@yeah.net

Keywords: breast cancer; cancer stem cell; SOX2 1-AS1; long non-coding RNA; SOX2

Received: October 20,2017 Accepted: December 04, 2017 Published: December 21, 2017

Copyright: Lu et al. This is an open-access article distributed under the terms of the Creative Commons Attribution License 3.0 (CC BY 3.0), which permits unrestricted use, distribution, and reproduction in any medium, provided the original author and source are credited.

\section{ABSTRACT}

Breast cancer stem cells (CSCs) are important element in the tumorigenesis and relapse. Long non-coding RNAs (IncRNAs) had been proved to regulate the breast cancer carcinogenesis. In present study, our research team aims to investigate the deepgoing role of SOX21-AS1 on breast CSCs properties and carcinogenesis. Human IncRNA microarray analysis and RT-PCR showed that SOX21-AS1 was up-regulated in breast cancer tissue. Moreover, the overexpression of SOX21-AS1 indicated the poor prognosis of breast cancer patients. Loss-of-function experiments revealed that SOX21-AS1 knockdown reduced the stem factors (Nanog, LIN28, Oct4 and SOX2) and CD44+/CD24- rate, suggesting the inhibitory role on breast CSC properties and selfrenewal ability. Besides, SOX21-AS1 knockdown inhibited the proliferation, invasion and tumor growth of breast cancer CSCs in vitro and in vivo. Bioinformatics online tools and luciferase reporter assay validated that miR-429 targeted SOX21-AS1 and SOX2 mRNA 3'-UTR, indicating the modulation of SOX21-AS1 and miR-429 on SOX2 protein expression. In summary, results conclude that IncRNA SOX21-AS1 modulates breast CSCs properties and carcinogenesis via targeting SOX2, providing a novel insight and therapeutic target for breast cancer.

\section{INTRODUCTION}

Breast cancer is one of the most common malignant tumors worldwide, acting as the leading cause of cancer-related death in female $[1,2]$. Among the breast cancer patients, nearly $20-40 \%$ of individuals suffer from neoplasm recurrence and tumor metastasis. The pathogenesis for breast cancer recurrence and metastasis is complex, involving multiple pathogenic factors and signaling pathway [3]. Besides, cancer cells have the ability to resist the chemotherapy drug, annotating as drug resistance. Cancer stem-like cells (CSCs) are a subpopulation of cancer cells with ability of initiating tumorigenesis, accounting for a small part in the tumor tissue, existing in many kinds of tumors including breast cancer [4]. CSCs are characterized by self-renewal and differentiation potential, which endow the potential of drug resistance, relapse and metastasis for cancer cells [5]. Increasing evidence have illustrated the critical role of CSCs in the osteosarcoma tumorigenesis, involved in differentiation, invasion and metastasis [6, 7].

Long non-coding RNAs (lncRNAs) are novel noncoding RNAs with more than 200 nucleotides in length and lack of transcription ability $[8,9]$. The function of IncRNAs on tumor cells stemness and CSC properties has been preliminarily investigated. For example, in human prostate cancer stem cells, lncRNA-ROR and Oct 4 mRNA both contain miR-145 binding sites and Oct4 and lncRNA-ROR directly compete for microRNA binding, activating the derepression of core transcription factors Oct4 [10]. In breast cancer, IncRNA SOX2OT is aberrantly over-expressed and led to an increased expression of SOX2, playing a key role in the maintenance of stem cell phenotypes [11]. LncRNA HOTAIR is highly 
upregulated in both CSC-MCF7 and CSC-MB231 cells and HOTAIR tightly regulates the proliferation, colony formation, migration and self-renewal capacity of CSCs, and upregulate Sox 2 expression, which is targeted by miR34a [12].

LncRNA SOX21-AS1 (SOX21 antisense RNA 1) is transcribed from SOX (SRYbox containing gene) intron. SOX2 has been verified as an important transcription factors and cancer stem-like cell marker in the tumorigenesis [13]. LncRNA SOX21-AS1 has been reported to be correlated with tumorigenesis [14]. In lung adenocarcinoma, the unusual overexpression levels of SOX21-AS1 is positively correlated with tumor size and advanced tumor-nodemetastasis (TNM) stage, and the inhibition of SOX21-AS1 remarkably induced $\mathrm{p} 57$ expression [15].

In present study, we focus on the role of IncRNA SOX21-AS1 on the cancer stem-like cells (CSCs) of breast cancer. Results reveal the function of SOX21-AS1 on breast cancer CSCs properties, proliferation and tumor growth through miR-429/SOX2 pathway.

\section{RESULTS}

\section{LncRNA SOX21-AS1 was over-expressed in breast cancer tissue and indicated poor prognosis}

In 3 pairs of breast cancer tissue and adjacent normal tissue, IncRNA microarray showed the aberrantly expressed lncRNAs (Figure 1A). Among these candidate IncRNAs, we noticed IncRNA SOX21-AS1, which was up-regulated in the microarray analysis. To verify the expression level of SOX21-AS1, RT-PCR was performed in breast cancer clinical samples (41 cases) (Figure 1B, Table 1) and cell lines (Figure 1E), showing the overexpression of SOX21-AS1. According to the median value of SOX21-AS1, breast cancer patients were divided into high expression level group and low expression level group (Figure 1C). Kaplan-Meier survival curves and log-rank tests were used to evaluate survival in all patients, showing the poor prognosis of breast cancer patients with high SOX21-AS1 expression (Figure 1D). Therefore, results conclude that lncRNA SOX21-AS1 was over-expressed in breast cancer tissue and cell lines, and indicated poor prognosis for breast cancer patients.

\section{LncRNA SOX21-AS1 knockdown inhibited the breast cancer CSC properties}

Breast cancer stem cells (CSCs) were performed for the further study, including CSC-MCF-7 and CSCMDA-MB-468. RT-PCR showed that SOX21-AS1 was up-regulated in CSC-MCF-7 and CSC-MDA-MB-468 (Figure 2A). Three short hairpin RNA (shRNA) were synthesized and transfected into CSC-MCF-7 and CSCMDA-MB-468 cells to knockdown SOX21-AS1 expression (Figure 2B). Sphere formation assays were performed (Figure 2C). Results showed that, in CSC-MCF-7 and
CSC-MDA-MB-468 cells, the number of primary spheres per 1000 cells and secondary spheres (P2) per 100 cells were both decreased (Figure 2D, 2E). RT-PCR showed that stem factors were down-regulated in SOX21-AS1 knockdown group, including Nanog, LIN28, Oct4 and SOX2 (Figure 2F). RT-PCR showed the relative expression level of SOX2 mRNA was upregulated in CSC-MCF-7 and CSC-MDA-MB-468 cells (Figure 2G). Flow cytometry showed that SOX21-AS1 knockdown decreased the $\mathrm{CD} 44^{+} /$ CD24- rate in CSC-MCF-7 and CSC-MDA-MB-468 subpopulation (Figure 2H, 2I). Overall, results indicated that SOX21-AS1 knockdown could inhibit the breast cancer CSC properties and self-renewal ability.

\section{SOX21-AS1 knockdown inhibited the proliferation and promoted apoptosis of breast cancer CSCs}

SOX21-AS1 had been verified to play vital role in properties and self-renewal ability. In this part, in vitro experiments were performed to test the role of SOX21AS1 knockdown on breast cancer CSCs proliferation and apoptosis. CCK-8 showed that SOX21-AS1 knockdown suppressed the proliferation vitality of CSC-MCF-7 and CSC-MDA-MB-468 cells compared with control group (Figure 3A, 3B). Colony formation assay showed that SOX21-AS1 knockdown decreased the clone number compared to negative cells (Figure 3C, 3D). Flow cytometry analysis showed that the apoptotic rate of CSC-MCF-7 and CSC-MDA-MB-468 cells was reduced by SOX21-AS1 knockdown (Figure 3E, 3F). Therefore, results indicated that SOX21-AS1 knockdown inhibited the proliferation and promoted apoptosis of breast cancer CSCs.

\section{SOX21-AS1 knockdown inhibited the invasion in vitro and tumor growth in vivo of breast cancer CSCs}

The role of SOX21-AS1 was tested using transwell invasion assay (Figure 4A). Results showed that SOX21AS1 knockdown inhibited the invaded CSC-MCF-7 and CSC-MDA-MB-468 cells compared with sh-NC group (Figure 4B). Xenograft assay in vivo was performed (Figure 4C). Results showed that SOX21-AS1 knockdown decreased the tumor volume (Figure 4D) and tumor weight (Figure 4E) in CSC-MCF-7 and CSC-MDAMB-468 cells. Besides, SOX21-AS1 expression level was decreased in knockdown group (Figure 4F). In summary, results indicated that SOX21-AS1 knockdown inhibited the invasion and tumor growth of breast cancer CSCs in vitro and in vivo.

\section{SOX21-AS1 regulated miR-429/SOX2 pathway}

Above results had indicated that SOX21-AS1 regulated the CSC properties, proliferation, tumor growth of breast cancer tumorigenesis. To explore the 
undergoing regulatory pathway of SOX21-AS1, we performed bioinformatics online tools to discover the target downstream molecular. Results showed that miR429 shared complementary binding sites with SOX21AS1 3'-UTR (Figure 5A). Luciferase gene reporter assay indicated that the luciferase intensity was decreased when SOX21-AS1 wild type combined with miR-429 mimics, suggesting the interaction within miR-429 and SOX21AS1 3'-UTR (Figure 5B). In breast cancer tissue, miR-429 expression level was down-regulated compared with non- tumor tissue (Figure 5C). Besides, miR-429 expression was up-regulated in CSC-MCF-7 cells transfected with sh-SOX21-AS1 (Figure 5D). Bioinformatics online tools showed that miR-429 targeted SOX2 mRNA 3'-UTR (Figure 5E). Luciferase gene reporter assay indicated that miR-429 combined with SOX2 mRNA 3'-UTR (Figure 5F). Western blot showed that SOX21-AS1 knockdown down-regulated the SOX2 expression, and miR-429 inhibitor downregulated the SOX2 expression (Figure 5G, 5H). Taken together, results revealed that
A

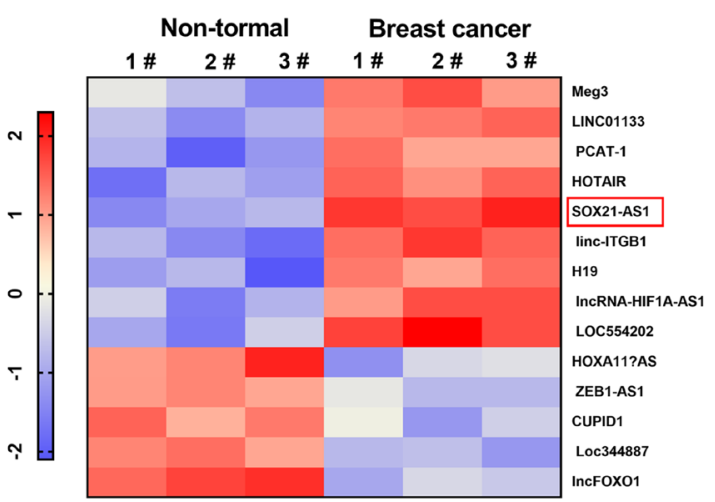

C

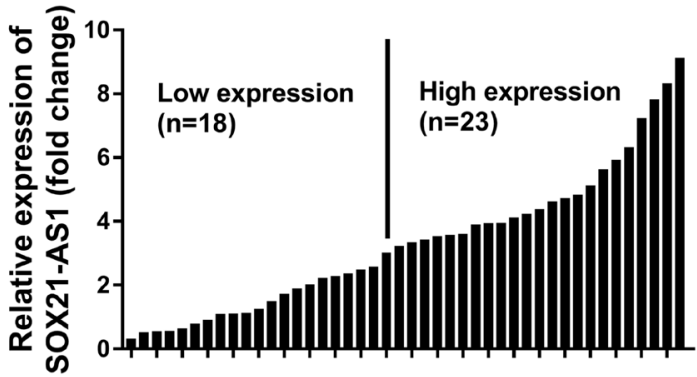

$\mathrm{E}$

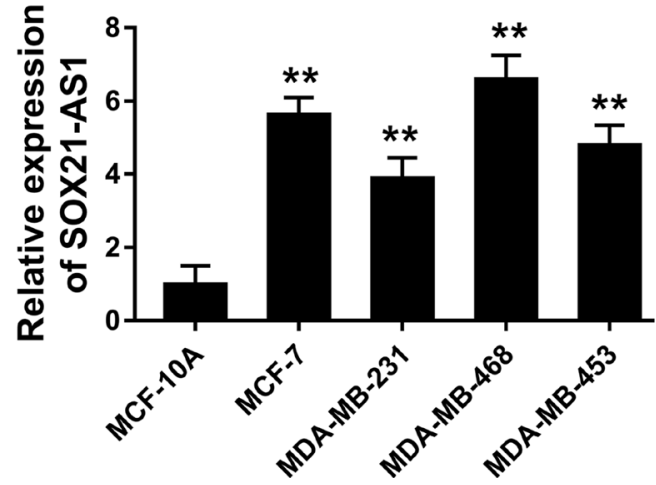

B

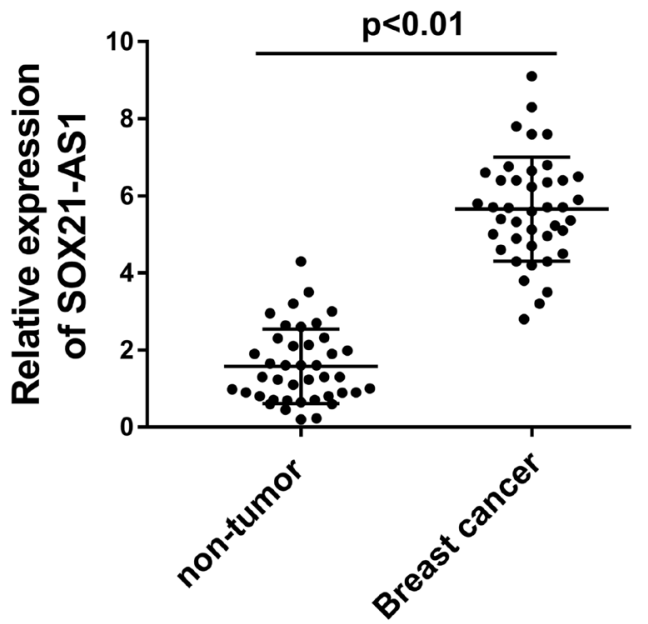

D

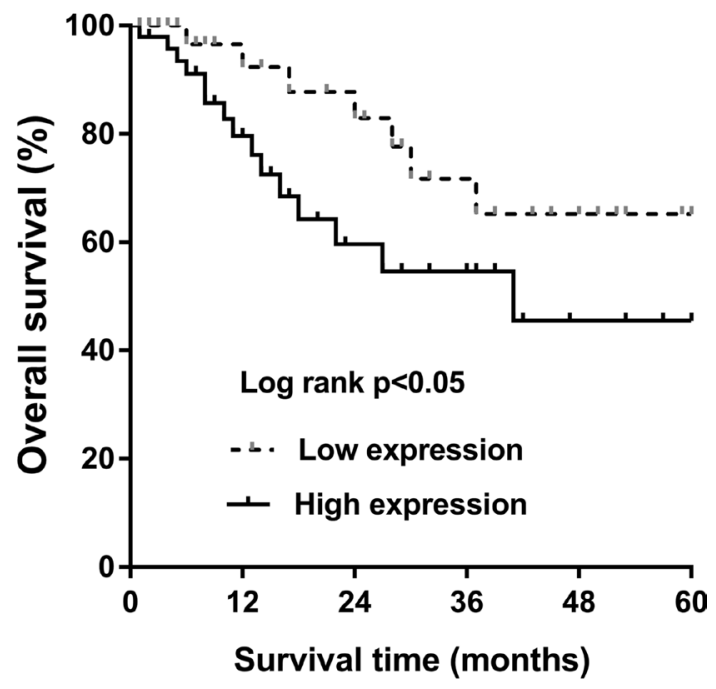

Figure 1: LncRNA SOX21-AS1 was over-expressed in breast cancer tissue and indicated poor prognosis. (A) LncRNA microarray analysis showed the aberrantly expressed lncRNAs in 3 pairs of breast cancer tissue and adjacent normal tissue. (B) RT-PCR showed the up-regulated expression of lncRNA SOX21-AS1 in 41 cases of breast cancer patients. (C) The distinction of breast cancer patients samples according to the SOX21-AS1 expression. (D) Kaplan-Meier survival curves and log-rank tests were used to evaluate the survival rate of breast cancer patients with high or low SOX21-AS1 expression. (E) RT-PCR showed the up-regulated expression of lncRNA SOX21-AS1 in breast cancer cell lines. ${ }^{* *} p<0.01$ presented significant difference. 
Table 1: Relationship between SOX21-AS1 expression and clinicopathological characteristics of breast cancer patients

\begin{tabular}{|c|c|c|c|c|c|}
\hline & & \multirow{2}{*}{$n=41$} & \multicolumn{2}{|c|}{ SOX21-AS1 } & \multirow{2}{*}{$\boldsymbol{P}$} \\
\hline & & & low, $n=18$ & high, $n=23$ & \\
\hline \multirow[t]{4}{*}{ type } & invasive ductal carcinoma & 32 & 15 & 17 & 0.149 \\
\hline & ductal carcinoma in situ & 6 & 2 & 4 & \\
\hline & mucinous adenocarcinoma & 2 & 1 & 1 & \\
\hline & Paget's disease & 1 & 0 & 1 & \\
\hline \multirow[t]{2}{*}{ age } & $<48$ years & 21 & 10 & 11 & 0.355 \\
\hline & $\geq 48$ years & 20 & 8 & 12 & \\
\hline \multirow[t]{2}{*}{ menopausal status } & premenopausal & 16 & 6 & 10 & 0.532 \\
\hline & postmenopausal & 25 & 12 & 13 & \\
\hline \multirow[t]{2}{*}{ ER status } & positive & 17 & 8 & 9 & 0.187 \\
\hline & negative & 24 & 10 & 14 & \\
\hline \multirow[t]{2}{*}{ distant metastases } & yes & 18 & 4 & 14 & $0.025^{*}$ \\
\hline & no & 23 & 14 & 9 & \\
\hline \multirow{2}{*}{$\begin{array}{l}\text { lymphatic } \\
\text { metastasis }\end{array}$} & positive & 20 & 7 & 13 & $0.011^{*}$ \\
\hline & negative & 21 & 11 & 10 & \\
\hline \multirow[t]{2}{*}{ TNM stage } & $\mathrm{I}-\mathrm{II}$ & 18 & 10 & 8 & $0.028^{*}$ \\
\hline & III-IV & 23 & 8 & 15 & \\
\hline
\end{tabular}

${ }^{*} P<0.05$ represents statistical differences. TNM, tumor-node-metastasis.

SOX21-AS1 knockdown down-regulated the SOX2 expression, presenting the positive regulation of SOX21AS1 on SOX2 expression through modulating miR-429.

\section{DISCUSSION}

Cancer stem-like cells (CSCs) are a subgroup of tumor cells with differentiative potential [20-22]. Breast cancer CSCs could generate neonatal cancer cells to accelerate the tumorigenesis and facilitate the cancer relapse [21, 23-25]. The role of long non-coding RNA (lncRNA) on breast cancer CSCs has been preliminarily investigated [26]. In present study, we try to explore the role of lncRNA SOX21-AS1 on breast cancer CSCs selfrenew and proliferation.

In present study, lncRNA SOX21-AS1 was found to be over-expressed in breast cancer tissue using both IncRNA microarray and RT-PCR. Moreover, the overexpression of SOX21-AS1 indicated poor prognosis of breast cancer patients. Therefore, our results conclude that SOX21-AS1 function as oncogenic molecular in the breast cancer oncogenesis. SOX21-AS1 is a new identified lncRNA, located at SOX (SRYbox containing gene) locus, which has been tested to regulate cancer tumorigenesis $[27,28]$. In oral squamous cell carcinoma, the aberrant promoter hypermethylation of SOX21-AS1 decreased the SOX21-AS1 expression and was significantly correlated with an advanced stage [14]. In lung adenocarcinoma, the higher expression levels of SOX21-AS1 positively correlated with tumor size and advanced TNM stage, and SOX21-AS1 knockdown significantly inhibited lung adenocarcinoma cells proliferation and metastasis [15]. Thus, the oncogenic role of SOX21-AS1 has verified once more in breast cancer.

The CSC properties are very vital for tumor growth, metastasis and relapse. The self-renewal ability of breast cancer CSCs makes it possible to accelerate the tumorigenesis. In present study, we found that SOX21AS1 knockdown decreased the number of primary spheres and secondary spheres, and reduced the stem factors (Nanog, LIN28, Oct4 and SOX2) and $\mathrm{CD}_{4} 4^{+} /$ $\mathrm{CD} 24^{-}$rate. All these indexes illustrate that SOX21-AS1 down-regulation could suppress the breast cancer CSCs properties and self-renewal ability. The inhibition of CSCs properties might significantly suppress the tumor growth.

In breast cancer cells, the subgroup of cells with $\mathrm{CD} 44^{+} / \mathrm{CD} 24^{-}$phenotype are considered to be enrich in CSCs. What's more, the subpopulation of cells has high degree of oncogenicity and invasion metastasis. In the progression of metastasis and relapse of breast cancer, the subpopulation breast cells play important roles. Balic M et al. (2017) found that the breast cancer cells with $\mathrm{CD}_{4} 4^{+} /$ $\mathrm{CD} 24^{-}$phenotype and CSC properties take important part in the early osseous metastasis [29, 30]. Debeb BG et al. (2010) found that histone acylation regulates $\mathrm{CD} 44^{+} /$ $\mathrm{CD} 24^{-}$breast cancer cells via $\mathrm{Wnt} / \beta$-catenin pathway [31]. Therefore, the decreasing of $\mathrm{CD} 44^{+} / \mathrm{CD} 24^{-}$rate by SOX21-AS1 knockdown proves the inhibition on breast CSC self-renewal ability. 
SOX2 is the most universally accepted stem cells marker, acting as the important gene for stem cells properties maintainance [32]. It has been verified that SOX2 is closely correlated with tumor occurrence and development $[33,34]$. In presents study, we found that
SOX21-AS1 targeted miR-429 and SOX2 mRNA. The expression of SOX21-AS1 is positively correlated with SOX2 protein expression. We conclude that SOX21-AS1 regulates the breast CSCs properties through miR-429 targeting SOX2 mRNA. Besides, SOX21-AS1 might
A

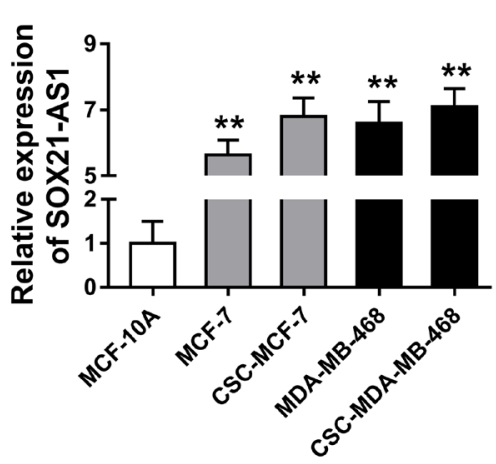

C

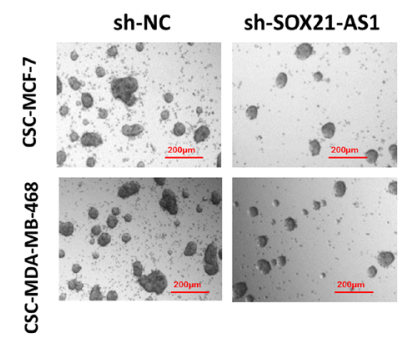

$\mathrm{F}$

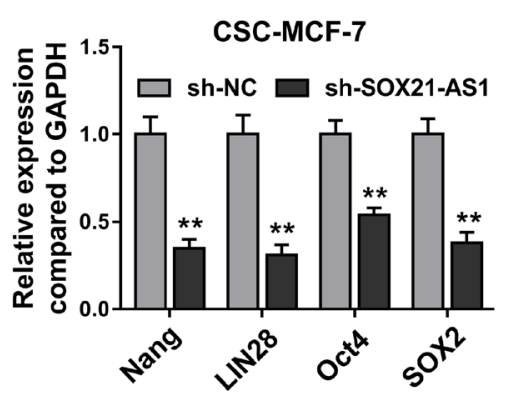

$\mathrm{H}$

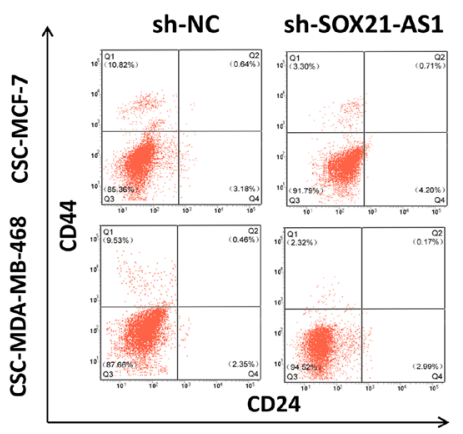

$\mathrm{B}$
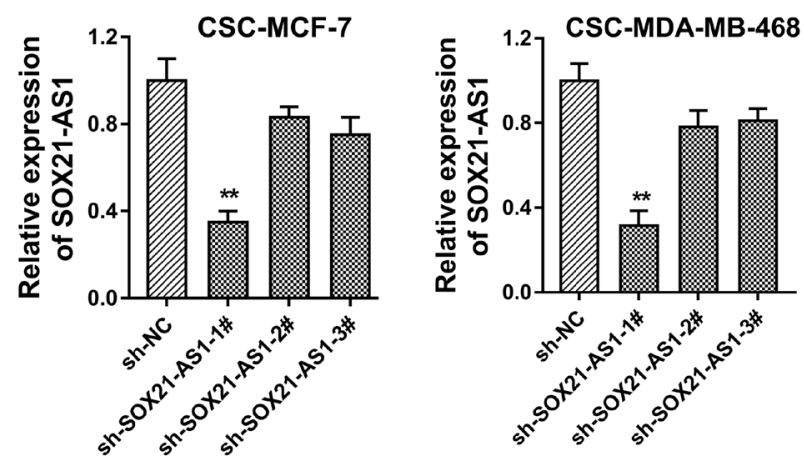

E

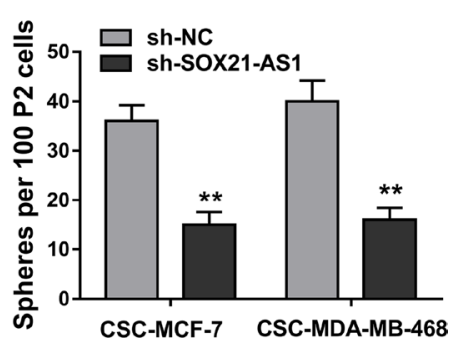

G

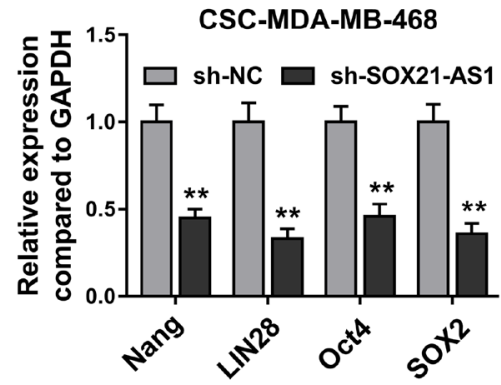

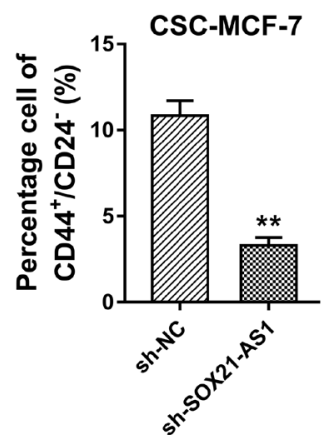

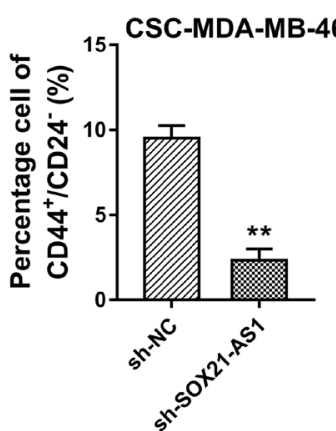

Figure 2: SOX21-AS1 knockdown could inhibit the breast cancer CSC properties and self-renewal ability. (A) RTPCR showed the SOX21-AS1 expression in CSC-MCF-7 and CSC-MDA-MB-468 cells. (B) Three short hairpin RNA (shRNA) were synthesized and transfected into CSC-MCF-7 and CSC-MDA-MB-468 cells. (C) Images of sphere formation assays. (D, E) Quantitative value of primary spheres per 1000 cells and secondary spheres (P2) per 100 cells. (F) RT-PCR showed the stem factors expression levels, including Nanog, LIN28, Oct4 and SOX2. (G) PCR showed the relative expression level of SOX2 mRNA in MCF, MDA-MB-468, CSCMCF-7 and CSC-MDA-MB-468 cells. (H, I) Flow cytometry showed the CD44 ${ }^{+} / \mathrm{CD}_{2} 4^{-}$rate in CSC-MCF-7 and CSC-MDA-MB-468 subpopulation. ${ }^{* *} p<0.01$ presented significant difference. 
also regulate other potential miRNAs and functional protein correlated with stemness, more than miR-429 and SOX2. The pathway of SOX21-AS1/miR-429/SOX2 is an important molecular mechanism. Zhou $\mathrm{M}$ et al. (2017) reported that IncRNA-Hh directly targets GAS1 to stimulate the activation of hedgehog signaling and increases the expression of GLI1, SOX2 and OCT4 to play a regulatory role in twist-positive breast cancer CSC maintenance [35].

In conclusion, our study investigates the expression and molecular role of IncRNA SOX21-AS1 on breast CSCs and tumorigenesis. Results validate the effective inhibition of SOX21-AS1 knockdown on breast CSCs properties and self-renewal ability, providing the important function of SOX21-AS1/miR-429/SOX2 pathway.

\section{MATERIALS AND METHODS}

\section{Tissue samples}

A total of 44 pairs of breast cancer samples and matched adjacent non-tumor samples were collected during surgery at Affiliated Cancer Hospital of Zhengzhou University, Henan Cancer Hospital between Jun 2014 and Jan 2016. None of patients had received antitumor treatment, chemotherapy and radiotherapy, before surgery.
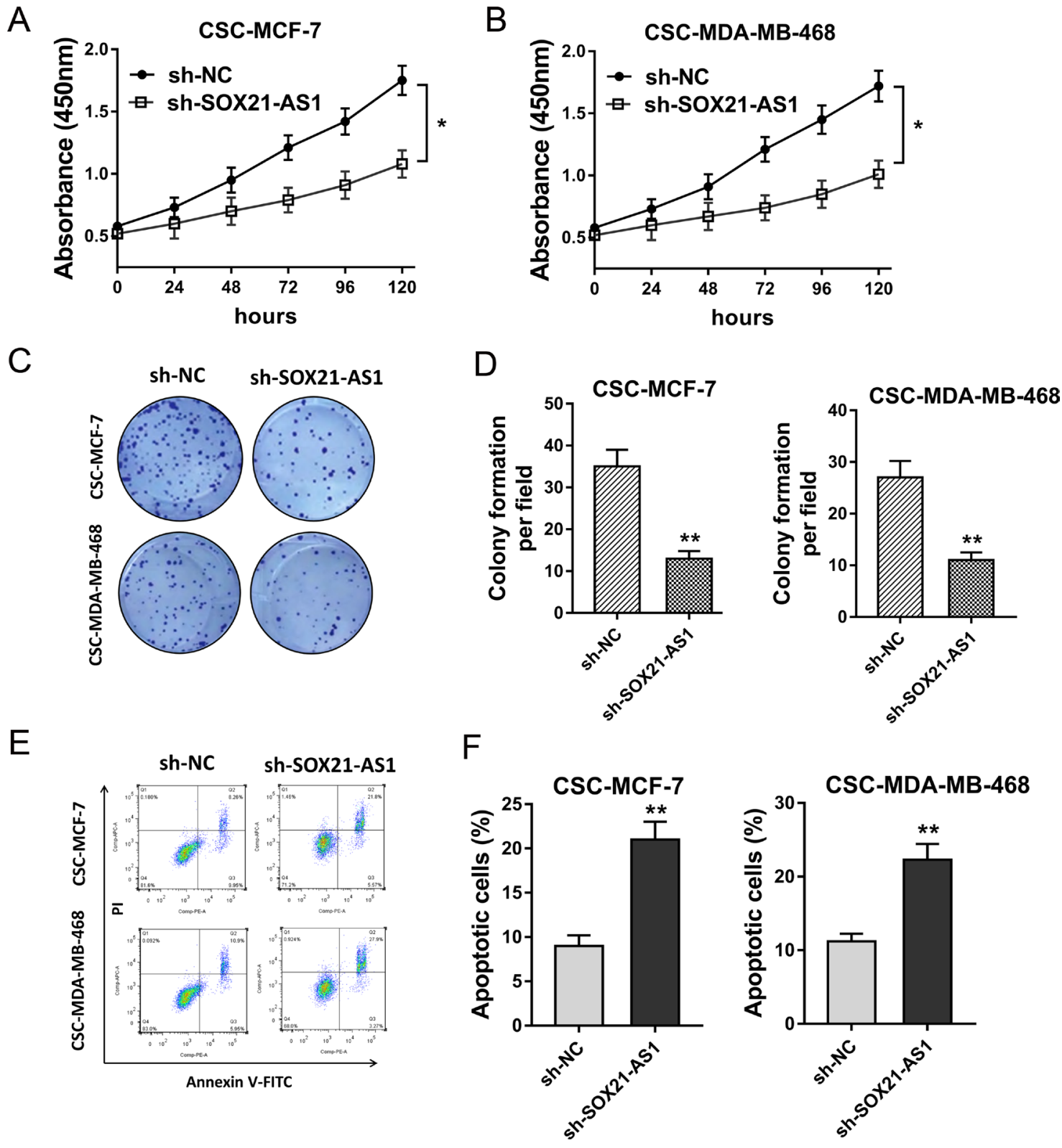

Figure 3: SOX21-AS1 knockdown inhibited the proliferation and promoted apoptosis of breast cancer CSCs. (A, B) CCK-8 showed the proliferation vitality of CSC-MCF-7 and CSC-MDA-MB-468 cells transfected with sh-SOX21-AS1 and shcontrol. (C) Images of colony formation assay. (D) Quantitative value of clone number. (E) Flow cytometry analysis showed the apoptotic rate of CSC-MCF-7 and CSC-MDA-MB-468 cells. (F) Quantitative value of apoptotic cells. ${ }^{*} p<0.05,{ }^{* *} p<0.01$ presented significant difference. 
The tumorous tissue was rapidly frozen at $-80^{\circ} \mathrm{C}$ for using. This study was approved by the Research Ethics Committee of Affiliated Cancer Hospital of Zhengzhou University, Henan Cancer Hospital. The informed written consents were collected from all patients.

\section{Cells culture and transfection}

Human breast cancer cell lines (MCF-7, MDAMB-231, MDA-MB-468, MDA-MB-453) and normal human breast epithelial cell (MCF-10A) were purchased from ATCC (Manassas, VA, USA). All cell lines were cultured in Dulbecco's modified Eagle's medium (DMEM,
Invitrogen, USA) supplemented with 10\% FBS (Gibco, Grand Island, NY, USA). All the cells were cultured at $37^{\circ} \mathrm{C}$ and $5 \% \mathrm{CO}_{2}$. The cancer stem-like cells, CSC-MCF-7 AND CSC-MDA-MB-468, were cultured and selected according previous literature [16-18]. For interference sequence transfection, oligonucleotides against SOX21AS1 (shSOX21-AS1) was synthesized by SBO Medical Biotechnology Company (Shanghai, China). The sequence of shSOX21-AS1 and negative control were shown as follows: shSOX21-AS1-1\#: 5'-CACCGGAGGGAAGAGATTCT GAAGATTCAAGTATCTCCTCCTTTTG-3', control: 5'-CA CCGTTCTCCGAACGTGTCACGTCAAGAGATTACGT GACACGTTCGGAGAATTTTTTG-3'. The transfection
A

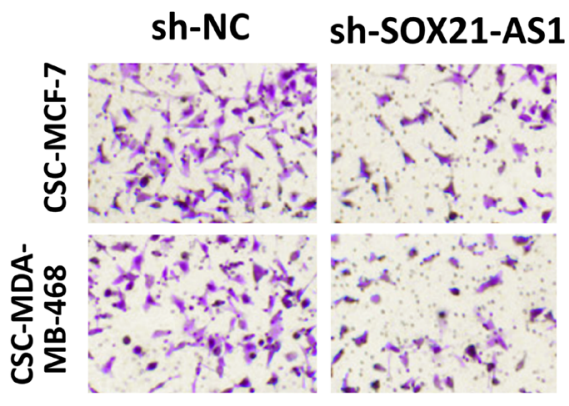

C

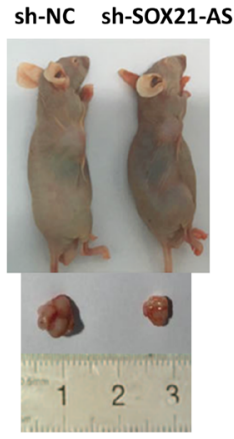

$E$

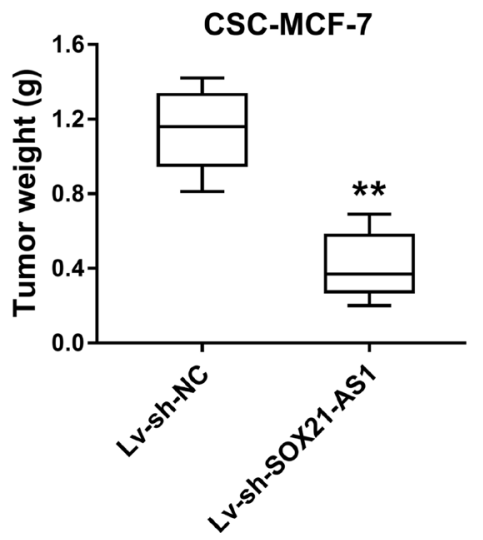

B
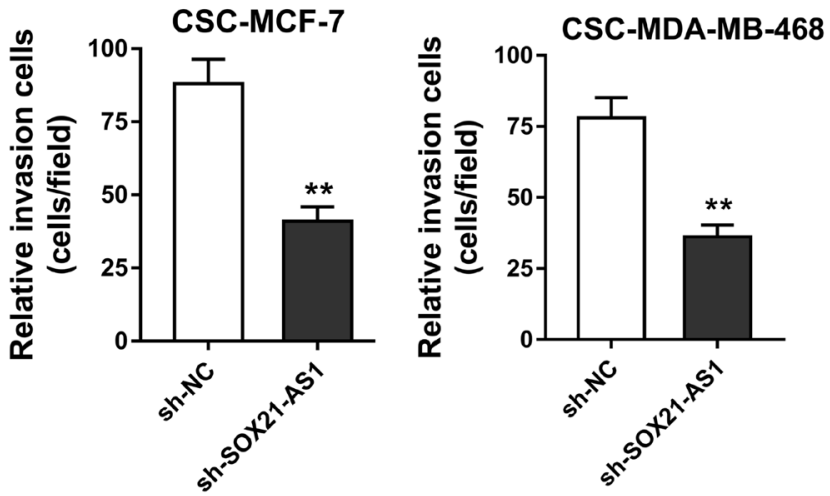
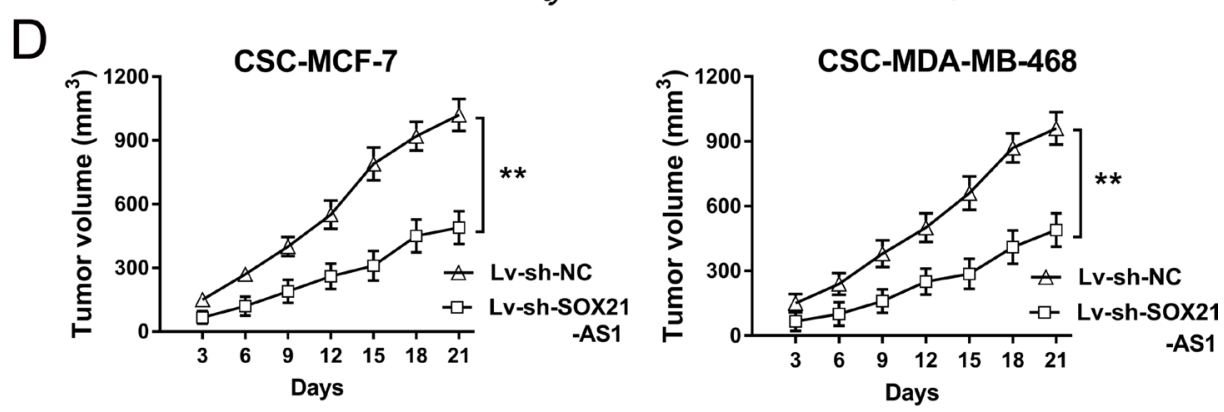

$\mathrm{F}$
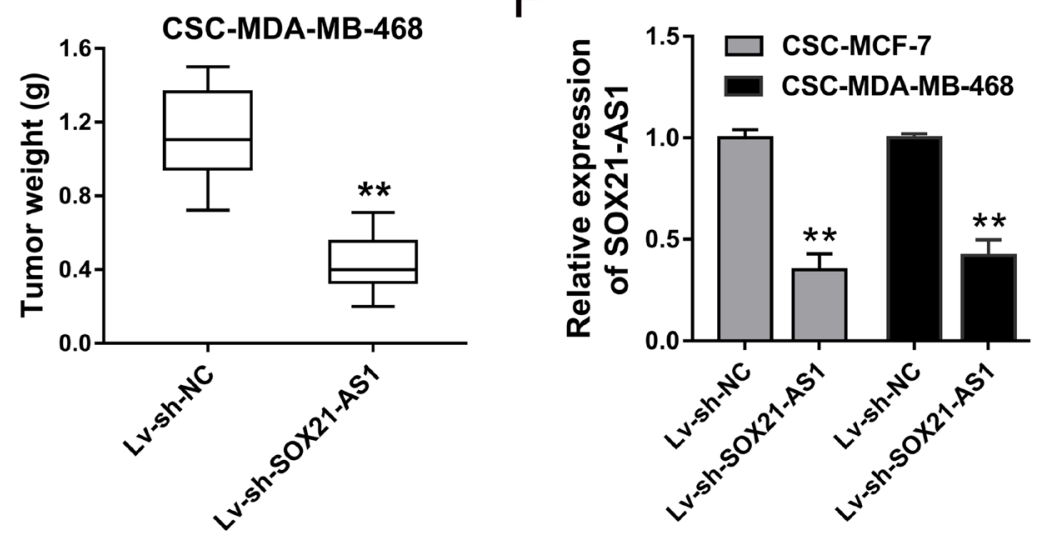

Figure 4: SOX21-AS1 knockdown inhibited the invasion in vitro and tumor growth in vivo of breast cancer CSCs. (A) Transwell invasion assay showed the invaded CSC-MCF-7 and CSC-MDA-MB-468 cells. (B) Quantitative data of invaded CSC-MCF-7 and CSC-MDA-MB-468 cells. (C) Photograph of xenograft mice assay in vivo. (D) Tumor volume of CSC-MCF-7 and CSCMDA-MB-468 cells growth measured every 3 days using formula: length $\times$ width $^{2} \times 0.5$. (E) Tumor weight of CSC-MCF-7 and CSCMDA-MB-468 cells. (F) RT-PCR showed the expression of SOX21-AS1. ${ }^{* *} p<0.01$ presented significant difference. 
details of lentivirus-mediated transfection were performed as previous reported [19]. For miR-429, the inhibitor and mimics sequences were chemically synthesized by Genechem (Shanghai, China) as follows: miR-429 mimics, forward, 5'-UGGCAGUGUCUUAGCUGGUUG-3', reverse, 5'-ACCAGCUAAGACACUGCCAUU-3'; miR-429 inhibitor, 5'-UUCUCCGAACGUGUCACGUUU-3'. The transfection was performed using Lipofectamine 2000 (Invitrogen) according to the manufacturer's instructions.

\section{LncRNA microarray analysis}

3 pairs of breast cancer tissue and adjacent normal tissue were used for lncRNA microarray analysis. Briefly, total RNA was extracted and human lncRNA microarray hybridization was performed according to Arraystar's standard protocols. The enriched RNA was amplified to
cDNA, and then transcribed into cRNA using Arraystar Super RNA Labeling Kit (Arraystar, Rockville, MD, USA). These labeled cDNA were hybridized using Affymetrix Human Transcriptome Array 2.0 (HTA 2.0) GeneChips (Affymetrix). Quantile normalization and subsequent data processing were performed using GeneSpringGX v. 11.0 software package (Agilent Technologies).

\section{Real time PCR}

Total RNA were extracted from tissue and cells using the Trizol reagent (Qiagen, Shanghai, China) according to the manufacture's instruction. cDNA was reversely synthesized using a HiScript ${ }^{\circledR}$ IIQRT SuperMix kit (Takara, Dalian, China) according to the manufacturer's instruction. RT-PCR was performed using the ABI PRISM 7000 Fluorescent Quantitative PCR System

A

SOX21-AS1 Mut 5' ... gatctgtaaatgaactacttgcat....3'

SOX21-AS1 Wild $5^{\prime}$....gatctgtaaatgatACAGTATTt... $3^{\prime}$ miR-429 $\quad$ 3'-TGCCAAAATGGUCTGTCAIIII
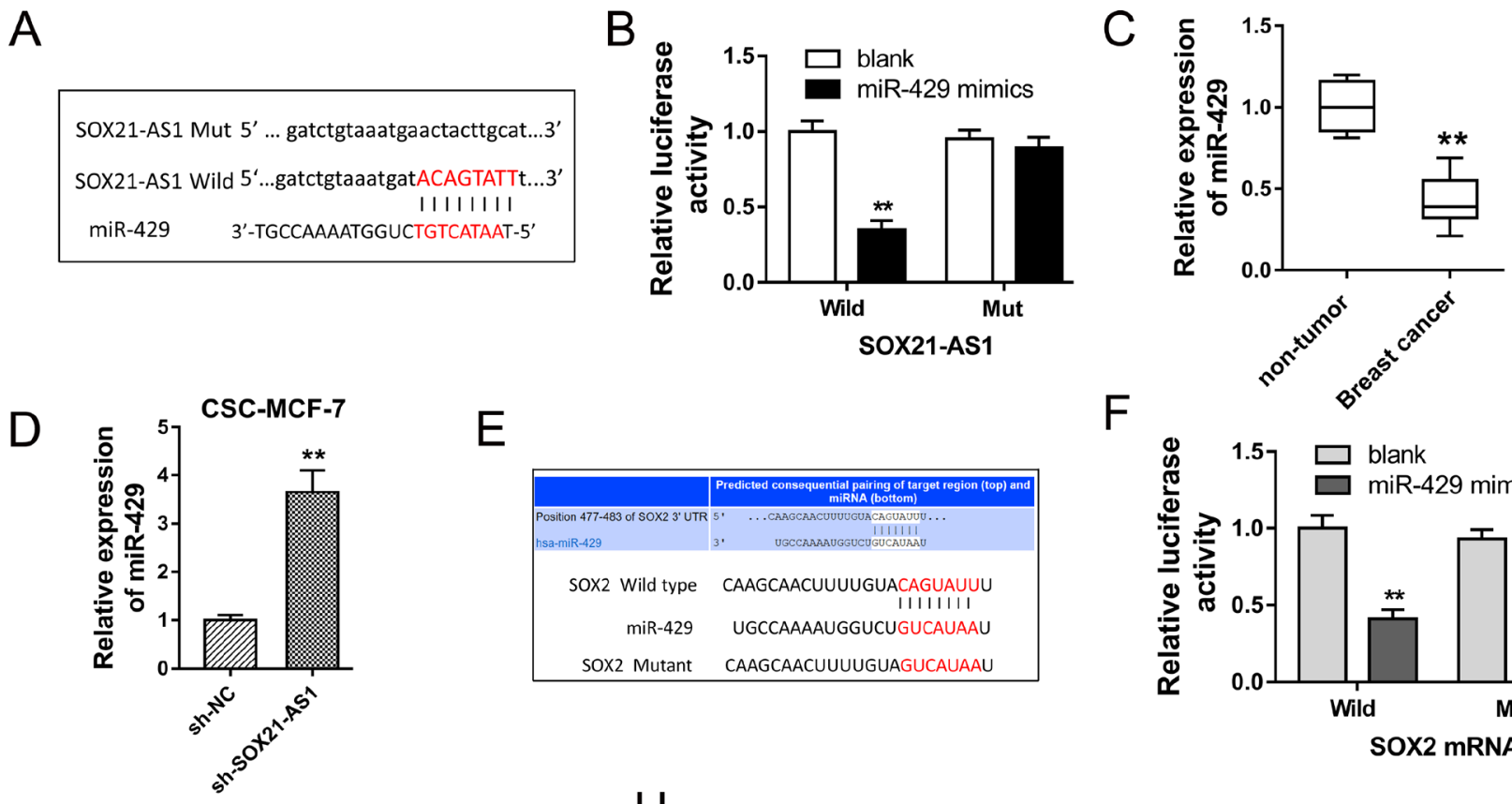

$\mathrm{F}$
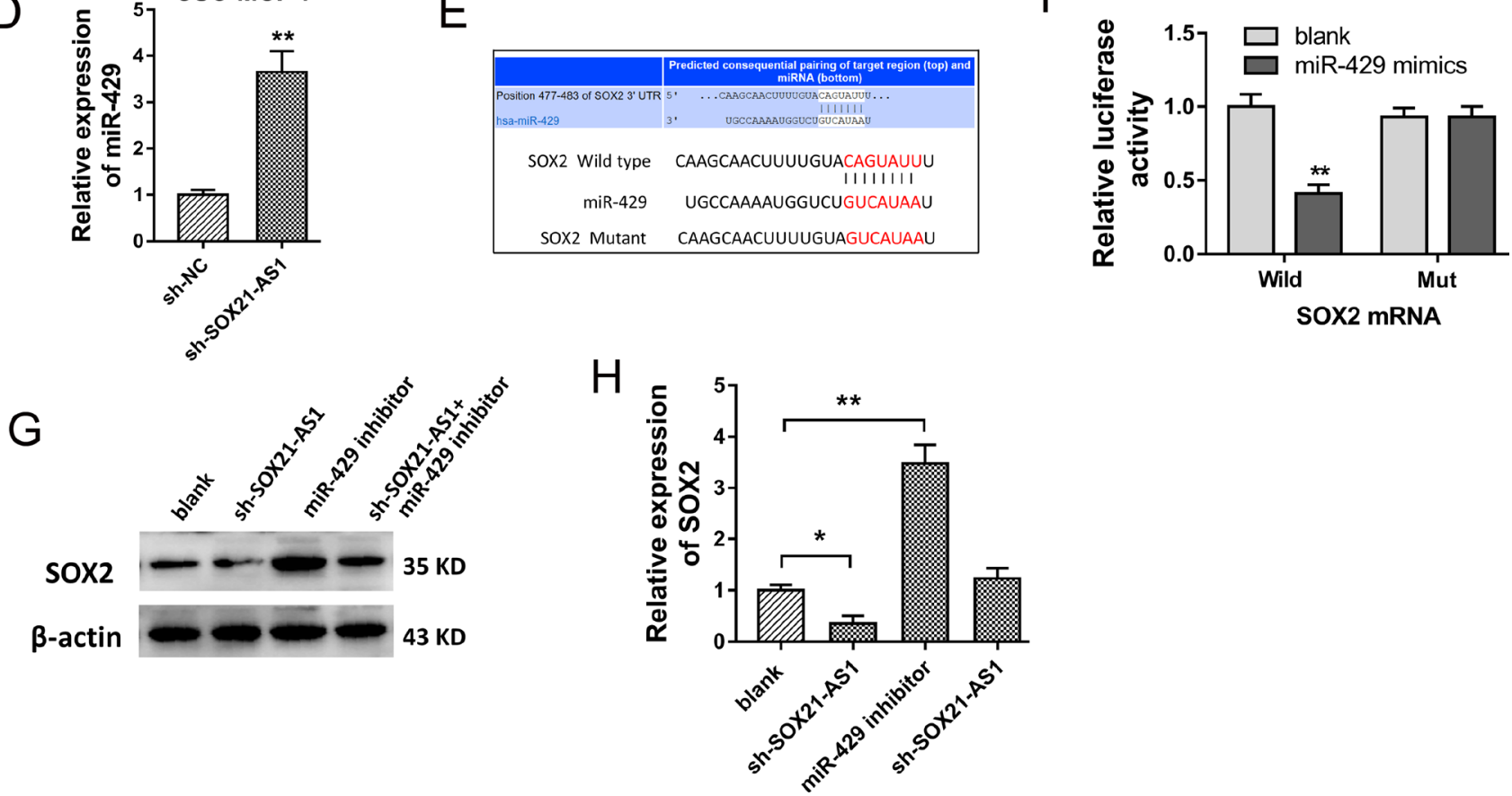

Figure 5: SOX21-AS1 regulated miR-429/SOX2 pathway. (A) Schematic diagram of SOX21-AS1 and miR-429 sequences, including SOX21-AS1 3'-UTR wild type and mutant type. (B) Luciferase gene reporter assay showed the interaction between SOX21-AS1 3'-UTR and miR-429. (C) RT-PCR showed miR-429 expression in breast cancer tissue. (D) RT-PCR showed miR-429 expression in CSCMCF-7 transfected with sh-SOX21-AS1. (E) Schematic diagram showed the sequences of miR-429 and SOX2 mRNA. (F) Luciferase gene reporter assay showed the interaction between miR-429 and SOX2 mRNA 3'-UTR. (G, H) Western blot showed the SOX2 expression. ${ }^{*} p<0.05,{ }^{* *} p<0.01$ presented significant difference. 
according to the manufacturer's instructions. The primer sequences in the study were shown as follows: SOX21AS1, forward, 5'-CGTGAAAGTGCCCCAATAGG TAGGTTCACA-3', reverse, 5'-TGGGGAATCTGTCTG TGTATCATTGGTT-3'; miR-429, forward 5'-GCAATA AGCGTCACGCTGTCTATG-3'; reverse, 5'-GTGGTTC GGGGTTAGACCGT-3'. GAPDH, forward, 5'-CCACATC GCTCAGACACCAT-3', reverse, 5'-ACCAGGCGCCCA ATACG-3'. Glyceraldehyde 3-phosphate dehydrogenase (GAPDH) was measured as an internal control for cell line. The Ct-value for each sample was calculated with the $2^{-\Delta \Delta \mathrm{Ct}}$ method.

\section{Sphere-formation assay}

Single breast cancer cell suspension $\left(3 \times 10^{3}\right.$ cells per ml) was plated in 6-well plates (Corning, Kraemer, CA, USA) added with modified DMEM. The first passage (P1) CSC-MCF-7 AND CSC-MDA-MB-468 cells were cultured and the spheres were counted after 14 days (passage one, P1). The secondary passage of CSCs were obtained in sphere formation assay. The spheres were reseeded in 96-well plates with 100 cells per well. After 10 days, the number of secondary spheres was counted.

\section{Flow cytometer}

CSC-MCF-7 and CSC-MDA-MB-468 were transfected with short hairpin RNA (shRNA) sh-SOX21AS1. Then, the apoptotic cell rate and $\mathrm{CD} 44^{+} / \mathrm{CD} 24^{-}$ cells were measured using flow cytometer analysis. Briefly, a total of $1 \times 10^{5}$ cells were resuspended in PBS containing $2 \%$ FBS and incubated with fluorescentlylabeled antibody: IgG1-pe/IgG-FITC, CD44-FITC/IgG1PE, CD24-PE/IgG2-FITC, CD44-FITC/CD24-PE. These antibodies were mixed in suspensions and incubated in the dark for $15 \mathrm{~min}$. Finally, cells were re-suspended in $500 \mathrm{ml}$ of FACS buffer and analyzed using a flow cytometer (BD Biosciences, Franklin Lakes, NJ, USA).

\section{Proliferation assay}

Cell proliferation was measured by CCK- 8 assay and colony formation assay. For the CCK-8 assay, CSCMCF-7 and CSC-MDA-MB-468 cells were seeded in 96-well culture plates with FBS-free medium for $12 \mathrm{~h}$. Then, the absorbance was detected at $450 \mathrm{~nm}$ after CCK-8 reagent (10 $\mu$, Dojindo, Japan) was added. For colony formation assay, CSC-MCF-7 and CSC-MDAMB-468 cells $\left(1 \times 10^{3}\right.$ cells per well $)$ were seeded into 6-well plate. After 14 days of culture, colony was fixed with $4 \%$ paraformaldehyde and stained with $0.1 \%$ crystal violet (Beyotime, Shanghai, China) for $30 \mathrm{~min}$. The colony number was counted under light microscopy. Every experiment was performed in triplicate.

\section{Transwell invasion assay}

The invasion of CSC-MCF-7 and CSC-MDAMB-468 using a transwell chamber assay (BD Biosciences, CA, USA) according to manufacturer's specification. Cells $\left(2 \times 10^{6}\right.$ cells $\left./ \mathrm{ml}\right)$ were treated with trypsinization and dissolved in DMEM without FBS. $1 \times 10^{5}$ cells/well in $200 \mathrm{~mL}$ of serum-free medium were added into the upper transwell chamber and $600 \mathrm{~mL}$ medium containing $20 \%$ FBS was added into the lower chamber. After incubation for $6 \mathrm{~h}$ at $5 \% \mathrm{CO}_{2}$ atmosphere at $37^{\circ} \mathrm{C}$, the invaded cells were fixed using $4 \%$ glutaraldehyde stained with $0.1 \%$ Crystal Violet. The cells number was counted under microscopy.

\section{Xenograft nude mice assay}

The xenograft animal studies were approved by the Institutional Animal Care and Use Committee of Affiliated Cancer Hospital of Zhengzhou University, Henan Cancer Hospital. Twenty male BALB/c nude mice (4 weeks, 8-10 g) were maintained under pathogen free conditions. CSCMCF-7 and CSC-MDA-MB-468 were transfected with lentivirus-mediated sh-SOX21-AS1 (Lv-sh-SOX21-AS1) or sh-NC (Lv-sh-NC) $\left(4 \times 10^{6}\right.$ cells/100 ul) and injected subcutaneously into the right side of the posterior flank of nude mice. Tumor volume was measured using caliper with $\left(\right.$ length $\times$ width $\left.^{2}\right) / 2$. Tumor weight was calculated after sacrifice.

\section{Dual-luciferase assay}

Full-length of SOX21-AS1 and SOX2 mRNA 3'-UTR sequence were amplified and cloned into psiCHECK-2 vector (Promega, Madison, WI., USA). Mutants and wild type within the miR-429 binding site were synthesized. Blank vectors and miR-429 mimics were co-transfected into HEK-293T cells with SOX21AS1 and SOX2 mRNA 3'-UTR sequence. Luciferase activity was measured using the Dual Glo Luciferase Assay System (Promega) following the manufacturer's instructions.

\section{Western blot}

Protein was extracted and lysed with ice-cold RIPA buffer (Sigma-Aldrich, USA), containing Tris$\mathrm{HCl}, 2-$ Mercaptoethanol, SDS and $10 \%$ glycerol. Protein concentration was confirmed by BCA Protein Assay Kit (Pierce Chemicals Co., USA). Protein samples were separated by $10 \%$ SDS-PAGE and transferred to polyvinylidene fluoride (PVDF) membranes. The membranes were blocked and incubated overnight at $4{ }^{\circ} \mathrm{C}$ with a primary antibody SOX2 (1:1000, Abcam), $\beta$-actin antibodies (1:500, Beyotime). Detection was performed with goat anti-rabbit IgG secondary antibody conjugated 
to horseradish peroxidase (Pierce) and an ECL detection kit (Beyotime Biotechnology, China).

\section{Statistical analysis}

All data were represented as mean \pm standard deviation (SD). Statistical analyses were calculated using SPSS 19.0 and diagrams were performed using GraphPad prism 5.0 software. Chi-square test was performed to analyze the correlation between clinicopathological characteristics. Univariate and multivariate Cox regression and Kaplan-Meier methods were used for survival analysis. Spearman's correlation was used for correlation analysis and Student's $t$-test was used for independent groups comparison. Statistical significance was considered as less than 0.05 .

\section{Author contributions}

Zhen-Zhen Liu designed the whole experiments and manuscript. De-Chuang Jiao, Jiang-Hua Qiao and Sen Yang assisted the major experiments. Zhen-Duo Lu performed the major work of the assay.

\section{ACKNOWLEDGMENT}

This work was supported by Henan Science and Technology Bureau Funding (132300410213).

\section{CONFLICTS OF INTEREST}

All authors declare no conflicts of interest

\section{REFERENCES}

1. Exner R. Local therapies for breast cancer. Memo. 2017; 10:181-4.

2. Niell BL, Freer PE, Weinfurtner RJ, Arleo EK, Drukteinis JS. Screening for Breast Cancer. Radiol Clin North Am. 2017; 55:1145-62.

3. Baltzer PAT, Kapetas P, Marino MA, Clauser P. New diagnostic tools for breast cancer. Memo. 2017; 10:175-80.

4. Chen Z, Qiu PY, Ma CG. Dexmedetomidine preconditioning protects against retinal ischemia/reperfusion injury and inhibits inflammation response via toll-like receptor 4 (TLR4) pathway. Biomed Pharmacother. 2017; 93:1018-24.

5. Zhao Z, Li S, Song E, Liu S. The roles of ncRNAs and histone-modifiers in regulating breast cancer stem cells. Protein Cell. 2016; 7:89-99.

6. Abarrategi A, Tornin J, Martinez-Cruzado L, Hamilton A, Martinez-Campos E, Rodrigo JP, Gonzalez MV, Baldini N, Garcia-Castro J, Rodriguez R. Osteosarcoma: Cells-ofOrigin, Cancer Stem Cells, and Targeted Therapies. Stem Cells Int. 2016; 2016:3631764.
7. Brown HK, Tellez-Gabriel M, Heymann D. Cancer stem cells in osteosarcoma. Cancer Lett. 2017; 386:189-95.

8. Gao YL, Zhao ZS, Zhang MY, Han LJ, Dong YJ, Xu B. Long Noncoding RNA PVT1 Facilitates Cervical Cancer Progression via Negative Regulating of miR-424. Oncol Res. 2017; 25:1391-8.

9. Li Q, Tian Y, Hu G, Liang Y, Bai W, Li H. Highly Expressed Antisense Noncoding RNA in the INK4 Locus Promotes Growth and Invasion of Renal Clear Carcinoma Cells via the beta-Catenin Pathway. Oncol Res. 2017; 25:1373-82.

10. Liu T, Chi H, Chen J, Chen C, Huang Y, Xi H, Xue J, Si Y. Curcumin suppresses proliferation and in vitro invasion of human prostate cancer stem cells by ceRNA effect of miR145 and lncRNA-ROR. Gene. 2017; 631:29-38.

11. Askarian-Amiri ME, Seyfoddin V, Smart CE, Wang J, Kim JE, Hansji H, Baguley BC, Finlay GJ, Leung EY. Emerging role of long non-coding RNA SOX2OT in SOX2 regulation in breast cancer. PLoS One. 2014; 9:e102140.

12. Deng J, Yang M, Jiang R, An N, Wang X, Liu B. Long Non-Coding RNA HOTAIR Regulates the Proliferation, Self-Renewal Capacity, Tumor Formation and Migration of the Cancer Stem-Like Cell (CSC) Subpopulation Enriched from Breast Cancer Cells. PLoS One. 2017; 12:e0170860.

13. Julian LM, McDonald AC, Stanford WL. Direct reprogramming with SOX factors: masters of cell fate. Curr Opin Genet Dev. 2017; 46:24-36.

14. Yang CM, Wang TH, Chen HC, Li SC, Lee MC, Liou HH, Liu PF, Tseng YK, Shiue YL, Ger LP, Tsai KW. Aberrant DNA hypermethylation-silenced SOX21-AS1 gene expression and its clinical importance in oral cancer. Clin Epigenetics. 2016; 8:129.

15. Lu X, Huang C, He X, Liu X, Ji J, Zhang E, Wang W, Guo R. A Novel Long Non-Coding RNA, SOX21-AS1, Indicates a Poor Prognosis and Promotes Lung Adenocarcinoma Proliferation. Cell Physiol Biochem. 2017; 42:1857-69.

16. Li B, Lu Y, Yu L, Han X, Wang H, Mao J, Shen J, Wang B, Tang J, Li C, Song B. miR-221/222 promote cancer stem-like cell properties and tumor growth of breast cancer via targeting PTEN and sustained Akt/NF-kappaB/COX-2 activation. Chem Biol Interact. 2017; 277:33-42.

17. Taube JH, Sphyris N, Johnson KS, Reisenauer KN, Nesbit TA, Joseph R, Vijay GV, Sarkar TR, Bhangre NA, Song JJ, Chang JT, Lee MG, Soundararajan R, et al. The H3K27me3demethylase KDM6A is suppressed in breast cancer stemlike cells, and enables the resolution of bivalency during the mesenchymal-epithelial transition. Oncotarget. 2017; 8:65548-65. https://doi.org/10.18632/oncotarget.19214.

18. Yang A, Qin S, Schulte BA, Ethier SP, Tew KD, Wang GY. MYC Inhibition depletes cancer stem-like cells in triplenegative breast cancer. Cancer Res. 2017; 77:6641-50.

19. Jiang N, Wang X, Xie X, Liao Y, Liu N, Liu J, Miao N, Shen J, Peng T. IncRNA DANCR promotes tumor progression and cancer stemness features in osteosarcoma 
by upregulating AXL via miR-33a-5p inhibition. Cancer Lett. 2017; 405:46-55.

20. Adamski V, Schmitt AD, Fluh C, Synowitz M, Hattermann $\mathrm{K}$, Held-Feindt J. Isolation and Characterization of FastMigrating Human Glioma Cells in the Progression of Malignant Gliomas. Oncol Res. 2017; 25:341-53.

21. Zhang F, Sun H, Zhang S, Yang X, Zhang G, Su T. Overexpression of PER3 Inhibits Self-Renewal Capability and Chemoresistance of Colorectal Cancer Stem-Like Cells via Inhibition of Notch and beta-Catenin Signaling. Oncol Res. 2017; 25:709-19.

22. Fang LZ, Zhang JQ, Liu L, Fu WP, Shu JK, Feng JG, Liang $\mathrm{X}$. Silencing of Btbd7 Inhibited Epithelial-Mesenchymal Transition and Chemoresistance in CD133+ Lung Carcinoma A549 Cells. Oncol Res. 2017; 25:819-29.

23. Audia A, Conroy S, Glass R, Bhat KPL. The Impact of the Tumor Microenvironment on the Properties of Glioma Stem-Like Cells. Front Oncol. 2017; 7:143.

24. Miyamoto S, Kanaseki T, Hirohashi Y, Tsukahara T, Kikuchi Y, Sato N, Torigoe T. Identification of cancer-stem cell antigens and development of CTL-mediated cancer immunotherapy. Nihon Rinsho Meneki Gakkai Kaishi. 2017; 40:40-7.

25. Lee SH, Hyun SK, Kim HB, Kang CD, Kim SH. Potential Role of CD133 Expression in the Susceptibility of Human Liver Cancer Stem-Like Cells to TRAIL. Oncol Res. 2016; 24:495-509.

26. Meng YB, He X, Huang YF, Wu QN, Zhou YC, Hao DJ. Long Noncoding RNA CRNDE Promotes Multiple Myeloma Cell Growth by Suppressing miR-451. Oncol Res. 2017; 25:1207-14.

27. Cheung LY, Okano H, Camper SA. Sox 21 deletion in mice causes postnatal growth deficiency without physiological disruption of hypothalamic-pituitary endocrine axes. Mol Cell Endocrinol. 2017; 439:213-23.
28. Moretto Zita M, Soncin F, Natale D, Pizzo D, Parast M. Gene Expression Profiling Reveals a Novel Regulatory Role for Sox 21 Protein in Mouse Trophoblast Stem Cell Differentiation. J Biol Chem. 2015; 290:30152-62.

29. Balic M, Schwarzenbacher D, Stanzer S, Heitzer E, Auer M, Geigl JB, Cote RJ, Datar RH, Dandachi N. Genetic and epigenetic analysis of putative breast cancer stem cell models. BMC Cancer. 2013; 13:358.

30. Tiran V, Stanzer S, Heitzer E, Meilinger M, Rossmann C, Lax S, Tsybrovskyy O, Dandachi N, Balic M. Genetic profiling of putative breast cancer stem cells from malignant pleural effusions. PLoS One. 2017; 12:e0175223.

31. Debeb BG, Zhang X, Krishnamurthy S, Gao H, Cohen E, Li L, Rodriguez AA, Landis MD, Lucci A, Ueno NT, Robertson F, Xu W, Lacerda L, et al. Characterizing cancer cells with cancer stem cell-like features in 293T human embryonic kidney cells. Mol Cancer. 2010; 9:180.

32. Garros-Regulez L, Garcia I, Carrasco-Garcia E, Lantero A, Aldaz P, Moreno-Cugnon L, Arrizabalaga O, Undabeitia J, Torres-Bayona S, Villanua J, Ruiz I, Egana L, Sampron $\mathrm{N}$, et al. Targeting SOX2 as a Therapeutic Strategy in Glioblastoma. Front Oncol. 2016; 6:222.

33. Huang J, Guo L. Knockdown of SOX9 Inhibits the Proliferation, Invasion, and EMT in Thyroid Cancer Cells. Oncol Res. 2017; 25:167-76.

34. Li S, Li B, Wang J, Zhang D, Liu Z, Zhang Z, Zhang W, Wang Y, Bai D, Guan J, Zhang Y. Identification of Sensitivity Predictors of Neoadjuvant Chemotherapy for the Treatment of Adenocarcinoma of Gastroesophageal Junction. Oncol Res. 2017; 25:93-7.

35. Zhou M, Hou Y, Yang G, Zhang H, Tu G, Du YE, Wen S, Xu L, Tang X, Tang S, Yang L, Cui X, Liu M. LncRNA-Hh Strengthen Cancer Stem Cells Generation in Twist-Positive Breast Cancer via Activation of Hedgehog Signaling Pathway. Stem Cells. 2016; 34:55-66. 